\title{
A Comparative Study of Charging Voltage Curve Analysis and State of Health Estimation of Lithium-ion Batteries in Electric Vehicle
}

\author{
Xuebing Han ${ }^{1}$ (i) $\cdot$ Xuning Feng ${ }^{1} \cdot$ Minggao Ouyang $^{1} \cdot$ Languang Lu$^{1} \cdot$ Jianqiu Li ${ }^{1} \cdot$ Yuejiu Zheng $^{2} \cdot \mathrm{Zhe} \mathrm{Li}^{1}$
}

Received: 30 May 2019 / Accepted: 4 November 2019 / Published online: 9 December 2019

(c) The Author(s) 2019

\begin{abstract}
Lithium-ion (Li-ion) cells degrade after repeated cycling and the cell capacity fades while its resistance increases. Degradation of Li-ion cells is caused by a variety of physical and chemical mechanisms and it is strongly influenced by factors including the electrode materials used, the working conditions and the battery temperature. At present, charging voltage curve analysis methods are widely used in studies of battery characteristics and the constant current charging voltage curves can be used to analyze battery aging mechanisms and estimate a battery's state of health ( $\mathrm{SOH}$ ) via methods such as incremental capacity (IC) analysis. In this paper, a method to fit and analyze the charging voltage curve based on a neural network is proposed and is compared to the existing point counting method and the polynomial curve fitting method. The neuron parameters of the trained neural network model are used to analyze the battery capacity relative to the phase change reactions that occur inside the batteries. This method is suitable for different types of batteries and could be used in battery management systems for online battery modeling, analysis and diagnosis.
\end{abstract}

Keywords Lithium-ion battery $\cdot$ Capacity fade $\cdot$ Charging voltage curve $\cdot$ Neural networks $\cdot$ Electric vehicle

\section{Introduction}

The energy crisis and environmental concerns have led to significant developments in electric vehicle technology and energy storage stations over the last few decades [1]. The Liion battery is one of the most critical components for energy storage because of its high energy and power density, long lifetime, and lack of a memory effect [2,3]. However, Li-ion cells degrade after repeated cycling [4]. The cell capacity fades and its resistance increases as a result, and this cell degradation affects the cell's energy storage ability and its output power capability. To improve the performance and reliability of the battery system, it is necessary to develop an appropriate battery management system (BMS). The BMS is required to estimate the battery state [5] and the battery's state of health $(\mathrm{SOH})$ in particular [6].

Minggao Ouyang

ouymg@tsinghua.edu.cn

1 School of Vehicle and Mobility, State Key Laboratory of Automotive Safety and Energy, Tsinghua University, Beijing 100084, China

2 College of Mechanical Engineering, University of Shanghai for Science and Technology, Shanghai 200093, China
Online SOH monitoring methods have been studied using a variety of tools and algorithms, and the related works have been reviewed in Refs. [7, 8]. These online $\mathrm{SOH}$ estimation methods can generally be summarized as the open-loop, the online observer, and the closed-loop estimation method. The open-loop estimation method is usually based on a battery aging model such as the Arrhenius aging model. The empirical model can be used to estimate battery $\mathrm{SOH}$ evolution based on the battery's working conditions, including the operating temperature, the charge/discharge rates, and the cutoff voltage [9-11]. The online observer method focuses on performance changes caused by aging and then estimates the battery's SOH using state observers such as a Kalman filter [12], a particle filter [13], or a neural network [14]. The closed-loop estimation method considers both the open-loop and the observer-based estimation results, making the results more stable $[15,16]$.

To build a battery aging model and estimate the $\mathrm{SOH}$ precisely, the battery aging mechanism must be studied in depth [17]. Repeated cycling of Li-ion batteries reduces the battery capacity via a number of aging mechanisms. These aging mechanisms may be related to the cathode and anode materials, the electrolyte, the cell separator, the operating conditions, and the working environment. 
The capacity fade rate may vary greatly under different aging mechanisms. For improved $\mathrm{SOH}$ estimation, diagnostics and battery health management, the battery capacity retention should be estimated and the battery aging mechanism, which influences the battery capacity, should be analyzed in the BMS $[18,19]$. Generally, battery aging can be caused by loss of lithium inventory (LLI) and active material (LAM), and incremental changes in resistance $[19,20]$. Based on the aging mechanism analysis results, the battery SOH could be analyzed more effectively and the BMS could then optimize the battery charging and discharging conditions to extend battery life [21-23].

Common battery aging mechanism measurement methods such as X-ray diffraction (XRD) and scanning electron microscopy (SEM) cannot be used in real BMSs in EVs because these methods involve the destruction of the batteries [24]. The aging mechanism can be reflected by the voltage characteristics of a battery in its open-circuit [17], charging [20], and discharging process [25]. These methods offer the potential to perform online battery diagnosis in the BMS [16]. In a real EV, the Liion batteries are usually charged via a constant current charging method through an onboard charger. Therefore, the change in the charging voltage curve after aging can be used to analyze the aging mechanism either inside the batteries without destruction of those batteries. There are already some appropriate voltage characteristic fitting and analysis methods, including methods based on polynomial functions [26], sigmoid function fitting [27], or fittings of other complex functions [28]. While voltage curve fitting is important, the results also indicate that analysis of the aging mechanism is very important and it has not been introduced in the previous studies.

Usually, the charging voltage curve can be analyzed using methods including IC analysis and differential voltage (DV) analysis. More information can be detected from the resulting IC and/or DV curves than from the charge curves alone [29, 30]. Methods involving IC curves have been introduced and validated by Dubarry [19, 31], Weng [32], and Safari [33], among others. Methods involving DV curves were introduced by Bloom [34, 35], Honkura [36], and Dahn [37]. However, the IC and DV curves can be used to find the internal changes and the degradation mechanism of Liion batteries during operation. IC curve analysis methods mainly focus on changes in the peaks and the valleys in the IC curve. However, to calculate both the IC curve and DV curve, voltage differentiation is needed, but it is usually difficult to obtain smooth and acceptable results.

Development of a reliable charging voltage curve analysis method for $\mathrm{SOH}$ monitoring of Li-ion batteries is focused to solve the above-mentioned problems. The contributions made in this paper are as follows:

(1) A novel method based on a neural network is developed to model the battery voltage characteristics.
(2) An IC curve calculation method is proposed based on the proposed neural network model, and the results show improved performance when compared with the traditional voltage differentiation method.

(3) Physical meanings related to the node parameters of the neural network are presented. Based on this theory, the aging mechanism and $\mathrm{SOH}$ of the battery are analyzed quantitatively.

When compared with the existing methods, the proposed method can be applied to fit the voltage characteristics with high stability. Changes in the voltage curve can be analyzed quantitatively to determine the aging mechanism and thus estimate the battery's $\mathrm{SOH}$. The proposed method is suitable for different battery types and can be used in the BMS for online battery modeling, analysis, and diagnostics.

It should be noted that this paper does not focus on the battery aging mechanism or the battery capacity loss characteristics versus the number of cycles. There are many factors and mechanisms that cause battery aging; this study primarily used the charging voltage curves during cell aging to investigate the aging mechanism via charging voltage curve analysis.

In Sect. 2, the experimental analysis of a commercial Li-ion cell is briefly introduced. In Sect. 3, the charging voltage curve and the IC curve of this cell are analyzed using the point counting method, the polynomial curve fitting method, and the proposed method based on the neural network model. The neural network takes the battery voltage as the input variable and the battery capacity as the output variable, and the neural network model is then trained based on the charging voltage curve. The fitting results, i.e., the parameters for each node in the trained neural network model, are then analyzed, and the IC curves can be calculated from the neural network model. A comparison, an analysis, and a discussion of the above methods are then presented in Sect. 4. Based on the neural network and the IC curve, the voltage plateaus related to the internal phase transformation reactions can be analyzed qualitatively using the node parameters. Then, based on the changes in the node parameters, the aging mechanism can be analyzed. Finally, the results from the proposed method are compared with the results from the traditional charging voltage curve analysis methods. Section 5 presents the conclusions drawn from the work.

\section{Battery Experiment}

Commercial prismatic $\mathrm{Li}$-ion cells were tested in this work. The cathode active material in these cells was $\mathrm{LiFePO}_{4}$ (LFP), and the anode active material was graphite. The 
nominal cell capacity according to the manufacturer is 6.5 A $\bullet$. This capacity value is used as the reference capacity to calculate the current rates (C-rates).

During the aging tests, the Li-ion cell was charged at $1 \mathrm{C}$ and discharged at $2 \mathrm{C}$ at a temperature of $50^{\circ} \mathrm{C}$. The cell charge cutoff voltage was $3.65 \mathrm{~V}$, and the discharge cutoff voltage was $2.00 \mathrm{~V}$. After every 90 cycles, a battery standard capacity test was conducted at room temperature $\left(25^{\circ} \mathrm{C}\right)$. The cell was fully charged by constant current charging of $1 / 2 \mathrm{C}$ to the charge cutoff voltage followed by constant voltage charging until the charge current decreased to $1 / 20 \mathrm{C}$; then, the battery was fully discharged by constant current discharging at $1 \mathrm{C}$ to the discharge cutoff voltage. The battery test procedure is illustrated in Fig. 1.

All charging and discharging tests were conducted using an eight-channel, UBT 100-020-8-type battery test bench (Digatron), which has a current range of -100 to $100 \mathrm{~A}$ and a voltage range of $0-20 \mathrm{~V}$. The voltage measurement accuracy is $1 \mathrm{mV}$. The battery test equipment is shown in Fig. 2 .

\section{Results and Charging Voltage Curve Analysis}

After 540 cycles, the battery capacity faded to approximately $90 \%$ of its initial capacity. The $1 / 2 \mathrm{C}$ constant current charging voltage curves obtained from the standard capacity tests after various numbers of cycles are shown in Fig. 3. During the constant current charging process, the cell voltage increased monotonically. The figure shows that there are three main voltage plateaus in the charging voltage curves. These voltage plateaus are mainly considered to be related to the phase transformation reactions of the Li-ion cell. The figure also clearly shows that the charging voltage curve changes significantly as the battery capacity

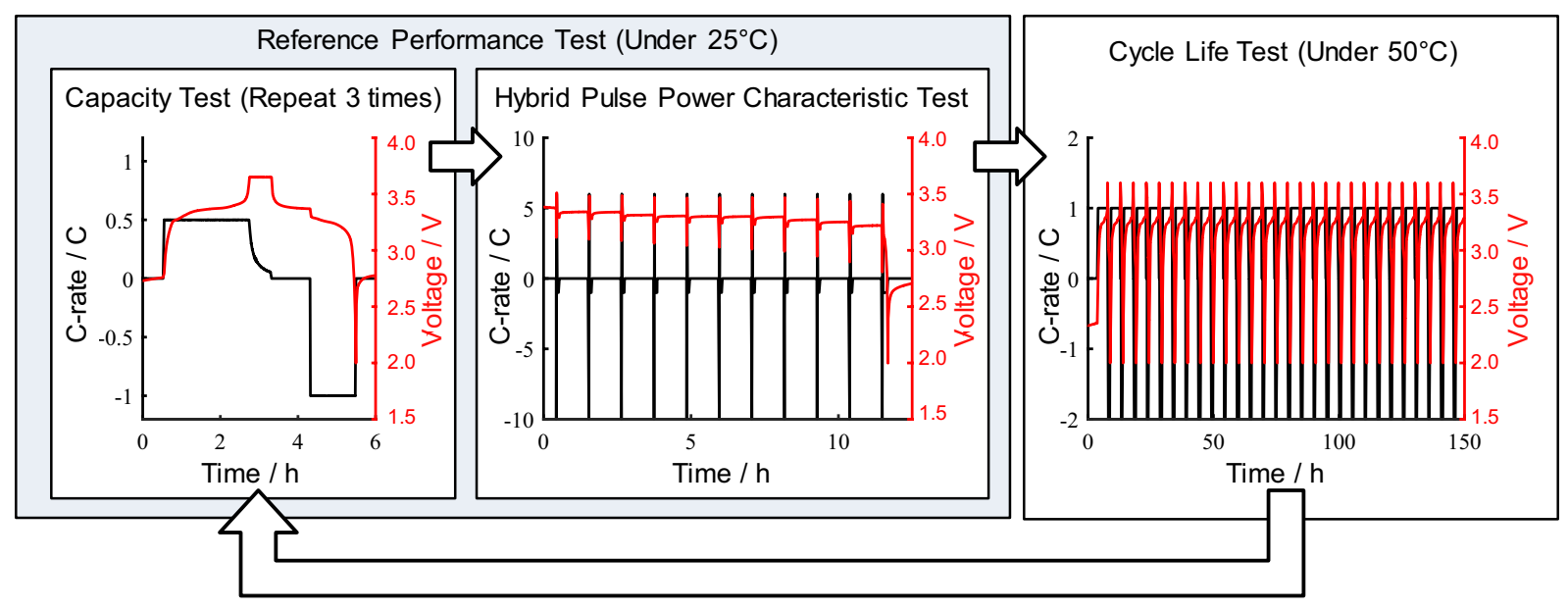

Fig. 1 Battery test procedure

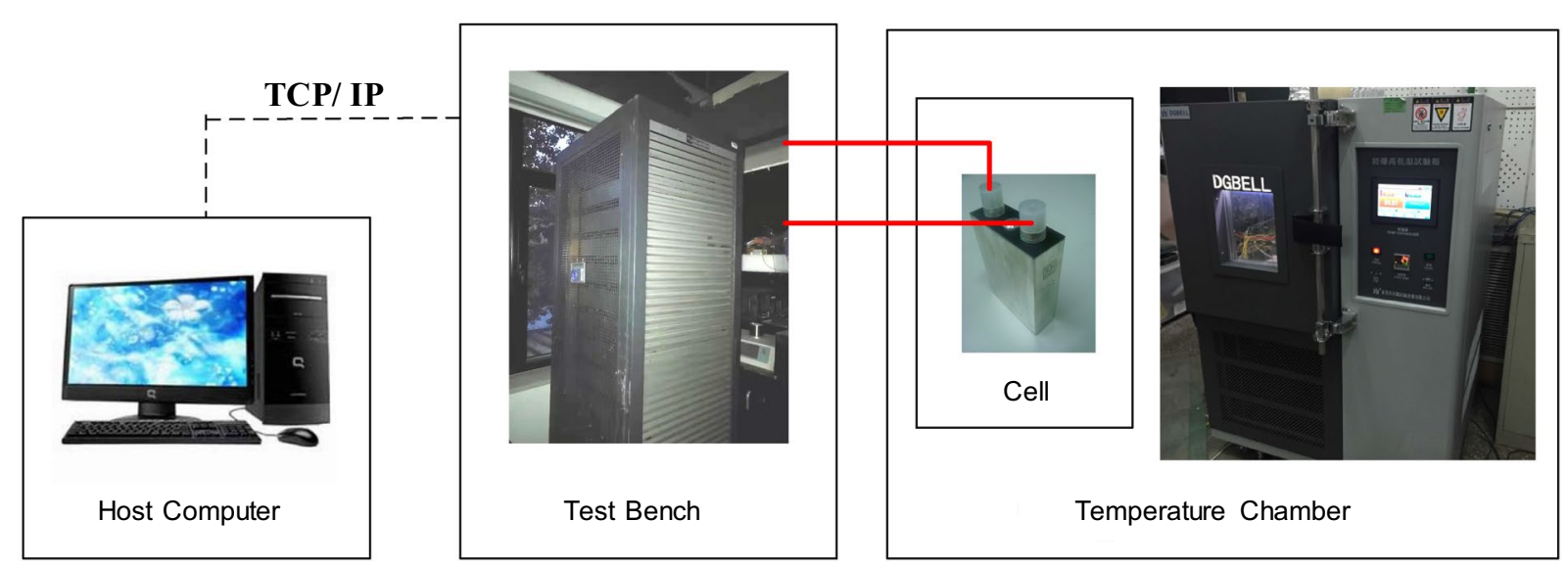

Fig. 2 Battery test equipment 


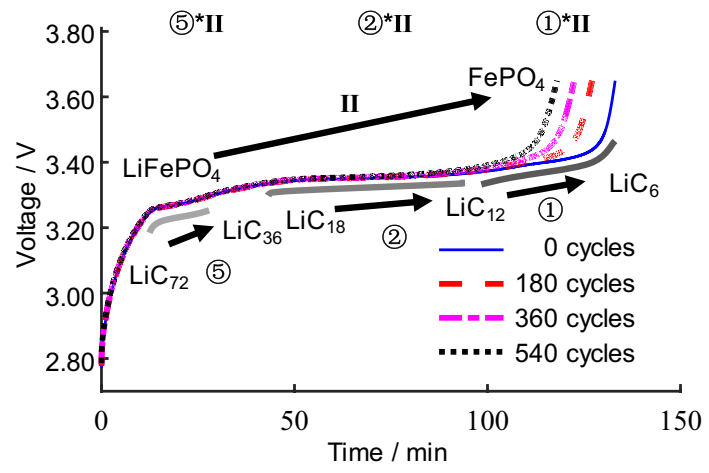

Fig. 3 Li-ion cell constant current charging voltage curves

fades. This change mainly occurs at the end of the constant current charging process, i.e., in the high state-of-charge (SOC) region, where the $3.40 \mathrm{~V}$ plateau gradually vanishes. In a real $\mathrm{EV}$, the Li-ion batteries are usually charged with a constant current charging method through a controllable charger, but are discharged using a dynamic discharging current based on the driving cycle and the driver's driving habits. The charging voltage curve could therefore possibly be obtained by the BMS and would be a better choice for use in analysis of the aging mechanism. In the next part of this paper, the $1 / 2 \mathrm{C}$ charging voltage curve is used to perform the analysis and modeling.

The IC curves $(\mathrm{d} Q / \mathrm{d} V)$ can be derived from the battery charging voltage curves. After differentiating, the voltage plateaus in the charging voltage curve can be transformed into clearly identifiable peaks in the IC curves. Therefore, the aging mechanism analysis based on the IC curves is simpler and more sensitive than that derived from the original voltage curves. The calculation of the IC curves is difficult and requires further discussion.

\subsection{Incremental Capacity Analysis Based on the Point Counting Method}

Numerical derivation of the charging voltage curve to obtain the IC curve is the most intuitive method for this analysis. To reduce the number of calculations and smoothen the results, a method based on the probability density function (PDF) was introduced in our previous work [38]. The PDF method needs to use the complete charging voltage curve to calculate the IC curve, and it can only be used to calculate the IC curve after the charging process is finished. For the online calculations to be performed in the BMS, an equivalent point counting method is used to calculate the IC curve here.

For the LFP cell under test in this work, the terminal voltage increases from approximately $2.80 \mathrm{~V}$ (depending on the depth of discharge) to $3.65 \mathrm{~V}$ during the constant current charging process. This voltage range can be divided into several voltage intervals. During the charging process, the battery voltage can be measured and the number of voltage points within each interval can be counted. In general, in a real EV, the constant current charging is adopted, while the voltage sampling frequency for the BMS is constant, usually approximately $1 \mathrm{~Hz}[5,39]$. The charged battery capacity is proportional to the number of sampling points during the charging process. This means that when the voltage increases at a higher rate, fewer points are present within the corresponding voltage interval, but when the voltage increases at a lower rate (i.e., when a voltage plateau occurs), more points are present within the corresponding voltage interval. Therefore, the IC curve (the $\mathrm{d} Q / \mathrm{d} V-V$ curve) can be calculated using Eq. (1):

$\frac{\mathrm{d} Q}{\mathrm{~d} V} \approx \frac{\Delta Q}{\Delta V}=\frac{n I}{3600 f \Delta V}$

where $n$ is the number of voltage points counted in the corresponding voltage interval, $I$ is the constant charging current, $f$ is the sampling frequency, $\Delta V$ is the width of each voltage interval, $V$ is the voltage and $Q$ is the capacity. The choice of $\Delta V$ influences the calculated results for the IC curve. The point counting method can calculate the IC curve during the charging process using a simple calculation process, and this method could be easily implemented in a real BMS. The IC curves for a fresh cell calculated using the point counting method for different $\Delta V$ values are shown in Fig. 4.

The figure shows that for a small value of $\Delta V$ (e.g., $1 \mathrm{mV}$ ), the IC curve varies significantly. The IC curve in this case can barely be used to analyze the aging mechanism because of the obvious noise in the results. In contrast, with a large value of $\Delta V$ (e.g., $10 \mathrm{mV}$ ), the IC curve is too smooth. In this case, the IC peak at the voltage of $3.40 \mathrm{~V}$ almost vanishes, so this IC curve cannot be used to analyze the aging mechanism either. Therefore, a $\Delta V$ value of $5 \mathrm{mV}$ is selected in the following part of this paper.

Additionally, there are three obvious peaks in the IC curves shown in Fig. 4 that correspond to the three voltage plateaus in the charging voltage curves. According to the electrochemical mechanism of LFP batteries, as a result of the insertion and extraction of the lithium ions, the cathode material gradually transforms from $\mathrm{LiFePO}_{4}$ into $\mathrm{FePO}_{4}$ or vice versa [40]. The cathode primarily goes through this $\mathrm{FePO}_{4}-\mathrm{LiFePO}_{4}$ phase transformation reaction during the battery charging and discharging processes. This cathode phase transformation reaction can be marked as II [31]. At the anode, with the insertion and extraction of the lithium ions, the anode material gradually transforms from $\mathrm{C}$ into $\mathrm{LiC}_{6}$ or vice versa. There are three obvious voltage plateaus, each represents a phase transformation process of the graphite anode, and the three corresponding phase transformation processes are denoted by (1), (2), and (5) [41]. Therefore, the three IC peaks, i.e., the three obvious voltage plateaus on the charging voltage curve for the 


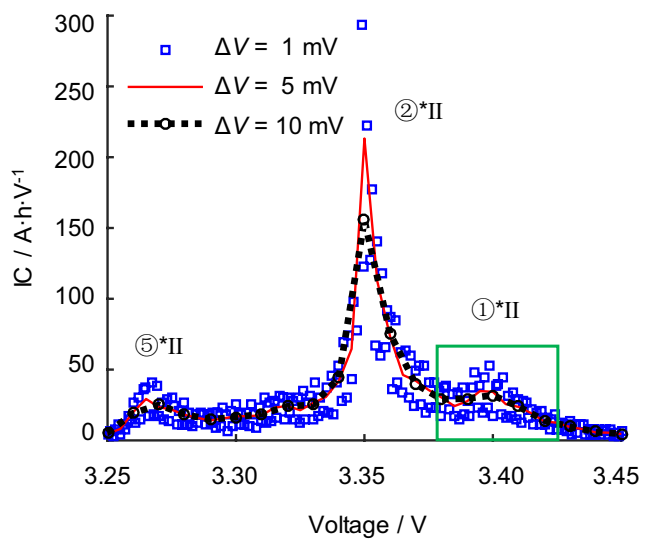

(a)

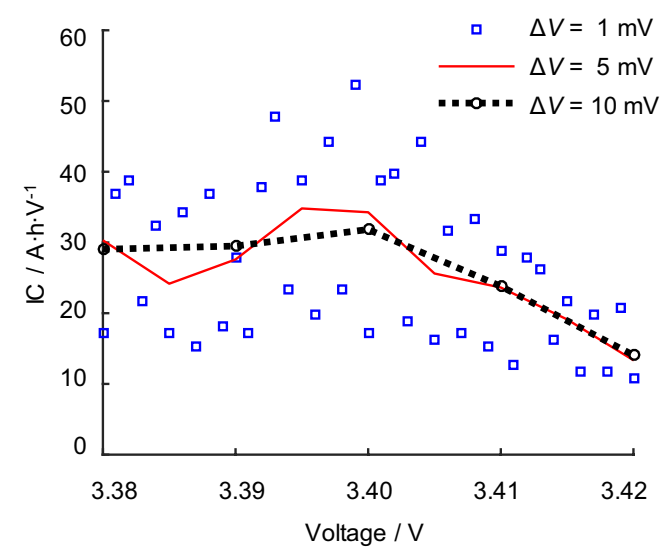

(b)

Fig. 4 IC curves for fresh cell with various $\Delta V$ values. a Complete IC curve and $\mathbf{b}$ magnified section of the derived IC curve

entire cell, represent the three-phase transformation processes of the graphite anode and the single-phase transformation process of the cathode. The three IC peaks can be labeled (1)*II, (2)*II, and (5)*II, as shown in Figs. 3 and 4.

It can easily be found that the area under each peak in the IC curve represents the capacity involved in the related reaction. The changes in the IC peaks represent the changes in the capacity related to the corresponding phase transformation reactions. The battery aging mechanism can thus be identified by analyzing the changes in each peak that appears with increasing cycle numbers.

\subsection{Incremental Capacity Analysis Based on the Polynomial Curve Fitting Method}

The point counting method is basically a numerical method that is used to obtain the IC curve. Because of the inevitable voltage measurement errors and noise, the IC curve results are usually very noisy when calculated directly with the point counting method. The choice of $\Delta V$ could therefore actually be considered to be a curve smoothing process. This smoothing process, however, may cause information loss. To solve these problems, some researchers have proposed methods based on curve fitting, such as the polynomial curve fitting method [42].

Figures 3 and 4 show that for the cell under test in this work, the cell voltage plateaus are mainly located between 3.20 and $3.45 \mathrm{~V}$. Therefore, the charging voltage curve within this voltage interval is used to perform the polynomial curve fitting process. The fitting function is shown in Eq. (2):

$V=f_{n}(Q)=p_{1} Q^{n}+p_{2} Q^{n-1}+\cdots+p_{n} Q+p_{n+1}$

where $n$ is the polynomial order number. The independent variable in this case is the capacity $Q$, and the dependent variable is the voltage $V$.

Then, based on the fitting results, the IC curve can be derived using Eq. (3):

$\frac{\mathrm{d} Q}{\mathrm{~d} V}=1 / f_{n}^{\prime}(Q)$

The polynomial order $n$ affects the IC curve results. If the polynomial order is low, the battery voltage curve cannot be described accurately, which causes huge errors in the IC curve; however, if the polynomial order is high, it may bring overfitting problems that cause unreasonable fluctuations in the IC curve. Therefore, a 16th order polynomial is selected in this paper.

A comparison of the experimental results and the fitting results for the fresh cell is shown in Fig. 5a, and the calculated IC curve is shown in Fig. 5b. The IC curve result is much smoother than the IC curve that was derived using the point counting method shown in Fig. 4. However, the calculations required for the polynomial curve fitting method are complex. In addition, the fitting results do not correspond directly to the related electrochemical mechanism as the capacities related to the IC peaks could not be obtained easily.

\subsection{Charging Voltage Curve Analysis Based on Neural Networks}

In general, IC analysis involves identifying the voltage plateaus (i.e., the phase transformation reactions) of the charging voltage curve and finding the capacities related to these voltage plateaus (i.e., the capacities related to the corresponding phase transformation reactions). Therefore, when the curve fitting method is adopted, an appropriate 


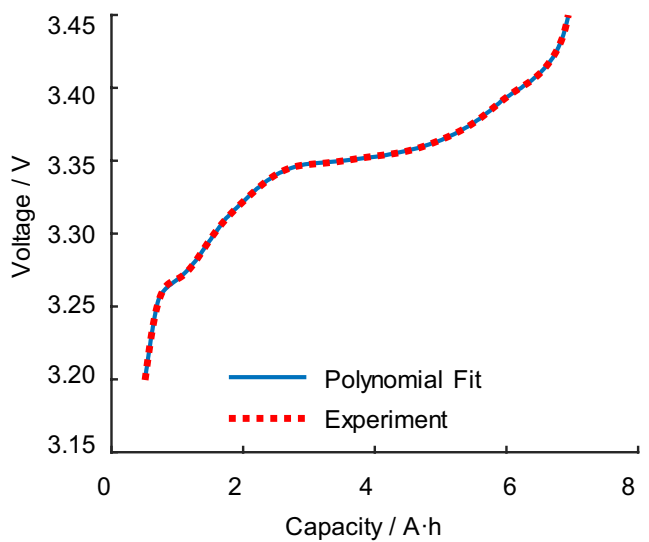

(a)

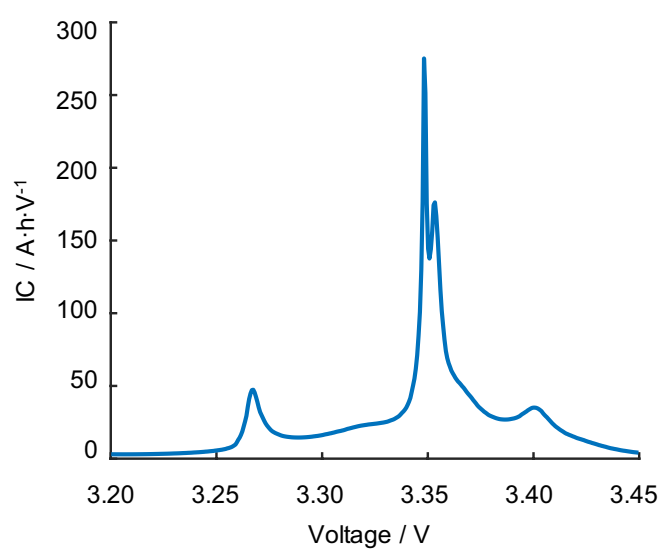

(b)

Fig. 5 IC curves for fresh cell derived using the polynomial curve fitting method. a Comparison of experiment results and fitting results and $\mathbf{b}$ derived IC curve

model that can express the voltage plateaus and the related capacities must be selected. In this section, a specific neural network model will be used to fit the battery charging voltage curves.

The neural network is widely used as a machine learning method because it is easy to be realized and simulated nonlinearly, and these networks have been used previously in battery management applications [14, 43, 44], particularly for estimation of battery issues involving aging. For example, a neural network method could be used to estimate the battery's SOH $[45,46]$, to estimate the battery's SOC during the degradation process [47] and to predict the remaining useful life of the battery [48].

Usually, the neural network is regarded as a "black box" model, which means that its internal parameters and processes are considered to be meaningless and to have little physical significance. The neural network is trained using large amounts of input and output data so that it is able to approximate various linear and nonlinear functions and fit the data precisely. The neural network parameters were not analyzed in the previous studies noted above. In this paper, the node parameters of the trained neural network model can reflect the capacity involved at each of the voltage plateaus directly.

As shown in Fig. 6a, a neural network usually contains at least three layers, comprising an input layer, a hidden layer, and an output layer. The hidden layer is usually formed using several nodes or, theoretically speaking, neurons. As shown in Fig. 6a, a single input, single output, and single hidden layer neural network model is proposed that takes the voltage as its input and the capacity as its output. This model is quite different from common models such as the polynomial model introduced in Sect. 3.2, which usually consider the battery capacity as the input and the voltage as the output.

A "neuron" in the hidden layer is a computational node that takes the input $V$ and a "+ 1 " intercept term and outputs $h_{w, b}$ as per Eq. (4):

$h_{w, b}=f(w V+b)$

where $w$ and $b$ are the node parameters that must be fitted, and the function $f$ is called the activation function. In this paper, the activation function is chosen to be the commonly used sigmoid function given by Eq. (5):

$f(t)=\frac{1}{1+\mathrm{e}^{-t}}$

The derivative of the sigmoid function then follows Eq. (6):

$f^{\prime}(t)=\frac{\mathrm{e}^{-t}}{\left(1+\mathrm{e}^{-t}\right)^{2}}$

The shape of the sigmoid function is shown in Fig. 6b, and the derivative of the sigmoid function is shown in Fig. 6c. The figures show that the sigmoid function presents a voltage plateau when its input is the voltage and its output is the capacity, i.e., $Q=f(V)$. The output (capacity) increases from 0 to 1 as the voltage increases. Additionally, the derivative of the sigmoid function shows a peak that corresponds to the voltage plateau. The area under this peak is 1 .

The output layer would then be set to be the weighted sum of the results from the neurons:

$Q=\sum w_{i}^{(2)} h_{w_{i}^{(1)}, b_{i}}+w_{0}^{(1)}=\sum \frac{w_{i}^{(2)}}{1+\mathrm{e}^{w_{i}^{(1)} V+b_{i}}}+w_{0}^{(1)}$

where $w_{i}^{(1)}$ represents the weight from the input layer to the $i$ th neuron, and $w_{i}^{(2)}$ represents the weight from the ith neuron to the output layer.

The charging voltage curve can then be fitted based on the neural network model described using Eqs. (4)-(7). 


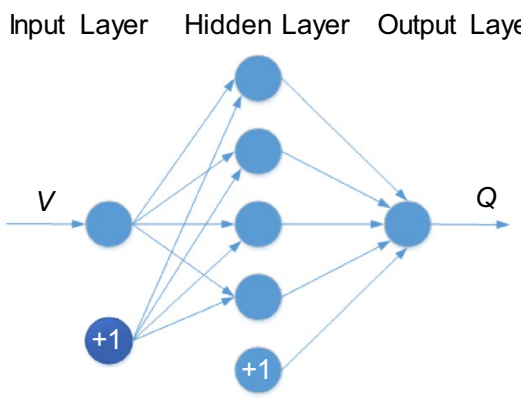

(a)

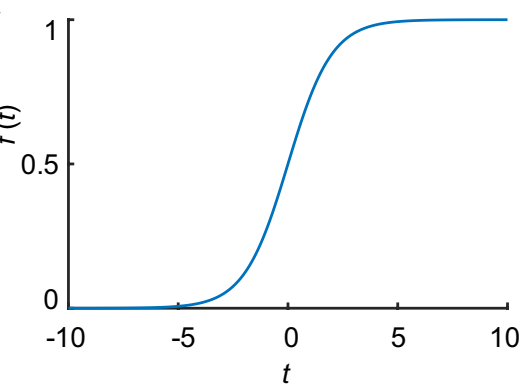

(b)

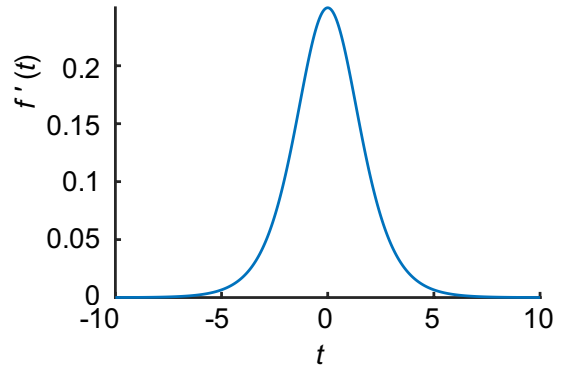

(c)

Fig. 6 Schematic diagram of a neural network. a Neural network model structure, $\mathbf{b}$ sigmoid function, and $\mathbf{c}$ derivative of sigmoid function

Here, voltage $V$ is selected as the input, capacity $Q$ as the output, and five nodes in the hidden layer in the neural network model. A larger number of nodes in the hidden layer may improve the model's accuracy but would significantly complicate the calculations, while fewer nodes would affect the fitting result error. The fitting results for a fresh cell are compared with the corresponding experimental results in Fig. 7a, and the same comparison of results for an aged cell is shown in Fig. 7b.

Because this model takes the voltage as its input and the capacity as its output, Fig. 7a, b is plotted with the voltage as the $x$-axis and the capacity as the $y$-axis. It should be noted that the experimental data shown in Fig. 7a and those shown in Fig. 5a are the same data, and only the $x$-axis and the $y$-axis have been interchanged. It can also be seen that the charging voltage curves were well fitted because of the excellent generalization ability of the neural network.

Based on the fitting results, the IC curve $(\mathrm{d} Q / \mathrm{d} V-V$ curve) could easily be calculated using Eq. (8). The IC curve can be considered to be the sum of $\mathrm{d} Q / \mathrm{d} V$ for each node from the neural network model:

$\frac{\mathrm{d} Q}{\mathrm{~d} V}=\sum w_{i}^{(2)} \frac{\mathrm{d} h_{w_{i}^{(1)}, b_{i}}}{\mathrm{~d} V}$

The derived IC curve for the fresh cell is shown in Fig. $7 \mathrm{c}$, and the corresponding curve for the aged cell is shown in Fig. 7d. The calculated IC curves are smooth, and the complete IC curve can be separated directly into several $\mathrm{d} Q / \mathrm{d} V$ curves based on the parameters for each node. Every node shown reflects a voltage plateau and the capacity related to this voltage plateau. Therefore, the capacity related to each voltage plateau can be derived quantitatively to enable aging mechanism identification.

\section{Discussion of the Neural Network Method}

\subsection{Comparison of IC Curves Derived by Different Methods}

The IC curves for a fresh cell that were derived using the different methods are compared in Fig. 8. In general, the IC curves calculated using the three methods described above show similar shapes. The three IC peaks can be found clearly in all the results above. The IC curve derived using the point counting method appears coarse. In addition, it is calculated directly from the experimental data, so this curve could be regarded as a reference IC result. The IC curve derived via the polynomial curve fitting method appears smoother, though there are some unreasonable fluctuations that may be caused by overfitting. The IC curve that was derived using the neural network method proposed in this paper appears to more closely approximate the IC curve derived using the point counting method, and the results are smoother and do not show the overfitting problem.

\subsection{Battery Aging Mechanism}

The IC curves calculated using different methods for cells after varying numbers of cycles are shown in Fig. 9. The results show that the IC curves derived by different methods show the same tendency for change. For the cell under test in this paper, all the peaks in the IC curve move slightly to the right, which means the internal cell resistance does not increase significantly with increasing numbers of cycles. Additionally, the heights of all the peaks decrease, which means that there is LAM inside the cell, which mainly comprises loss of the anode material. The results also show that the peak (1)*II decreases more significantly than the other peaks, which means that LLI is occurring inside the LFP 


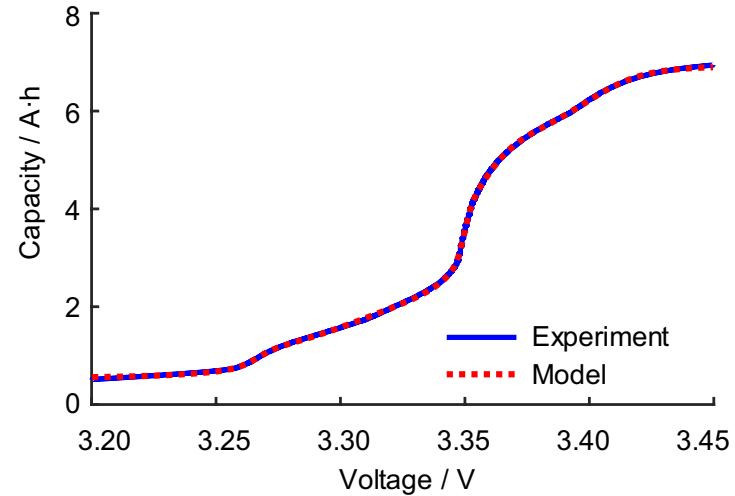

(a)

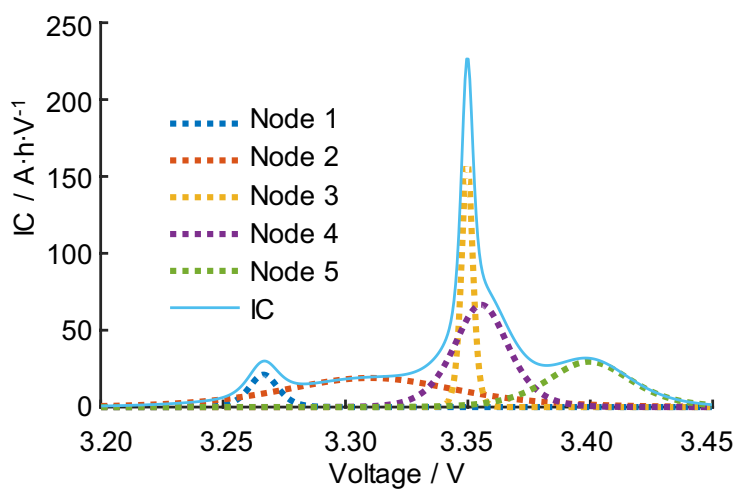

(c)

Fig. 7 Neural network fitting results and calculated IC curves. a Comparison of experimental results and fitting results for fresh cell. b Comparison of experimental results and fitting results for aged

cell, and this may be the main aging mechanism. Results in the literature $[31,33,49]$ also led to the same conclusion about the aging mechanism of commercial LFP cells based on aging experiment results, indicating that LLI is the main mechanism behind the fade in the LFP cell capacity and that the LAM of the anode may also affect the capacity.

\subsection{Quantitative Analysis of Charging Voltage Curve}

The IC analysis usually involves calculating the IC curves and comparing the location and height of each peak qualitatively. When derived using the numerical derivation method or the polynomial curve fitting method, the heights of the IC peaks can be used to analyze the battery aging quantitatively, but the heights of the peaks have little physical meaning. The capacities related to the peaks that represent the Li-ions involved in the corresponding phase transformation

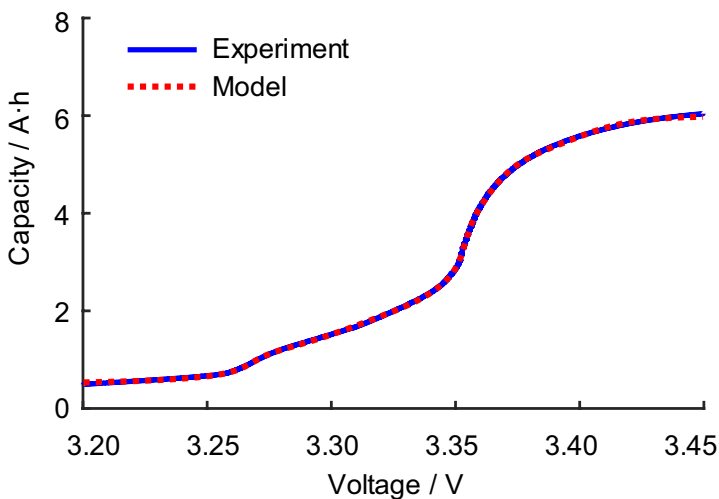

(b)

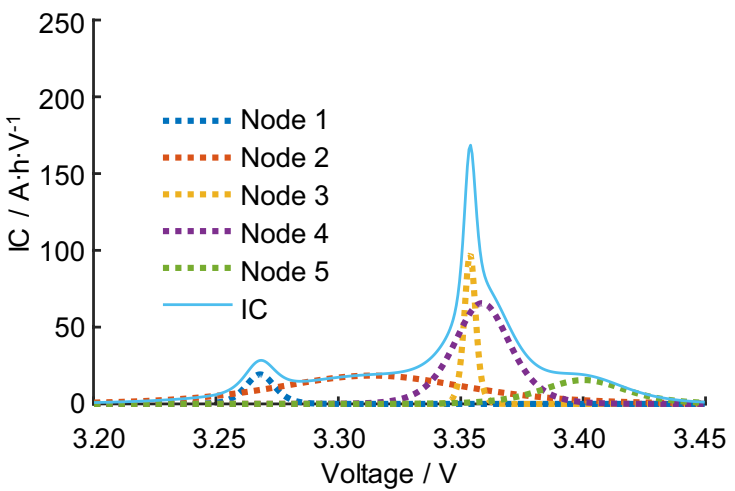

(d)

cell after 540 cycles. c Derived IC curve for fresh cell. d Derived IC curve for aged cell after 540 cycles

reactions have clearer physical meanings and can thus be used to analyze the battery aging condition quantitatively.

As shown in Fig. 7c, d, when calculated based on the neural network model, the peaks of the IC curves can be separated into the sums of the $\mathrm{d} Q / \mathrm{d} V$ curves for each node, as shown in Eq. (8). Then, based on the parameters for each node, the capacity related to each voltage plateau can be analyzed quantitatively.

Generally, the $\mathrm{d} Q / \mathrm{d} V$ curve for a typical node in the neural network model presents symmetrical peaks, as shown in Fig. $6 c$ and by Eq. (8). The shape of each peak is affected by $w_{1}^{(1)}$, while the location of each peak is calculated using the corresponding voltage plateau $E_{0}$, which can be calculated using Eq. (9):

$$
E_{0, i}=-\frac{b_{i}}{w_{i}^{(1)}}
$$




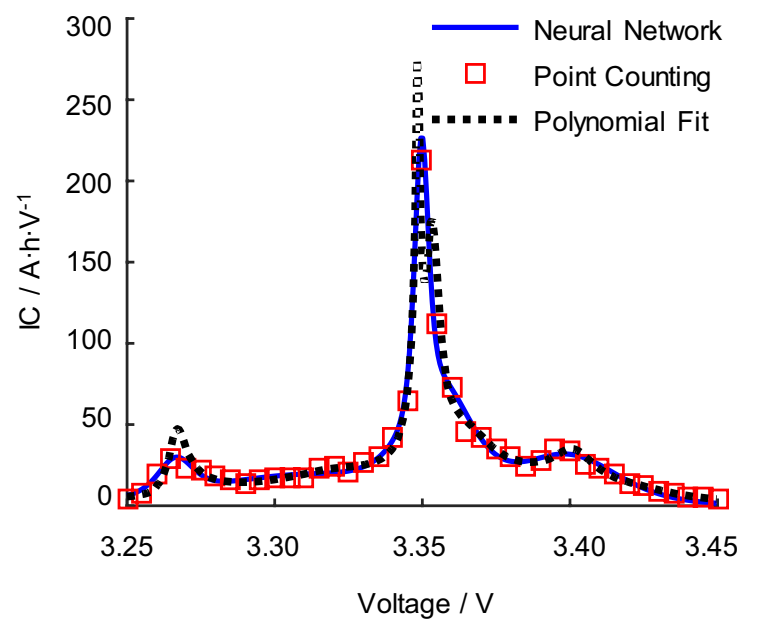

(a)

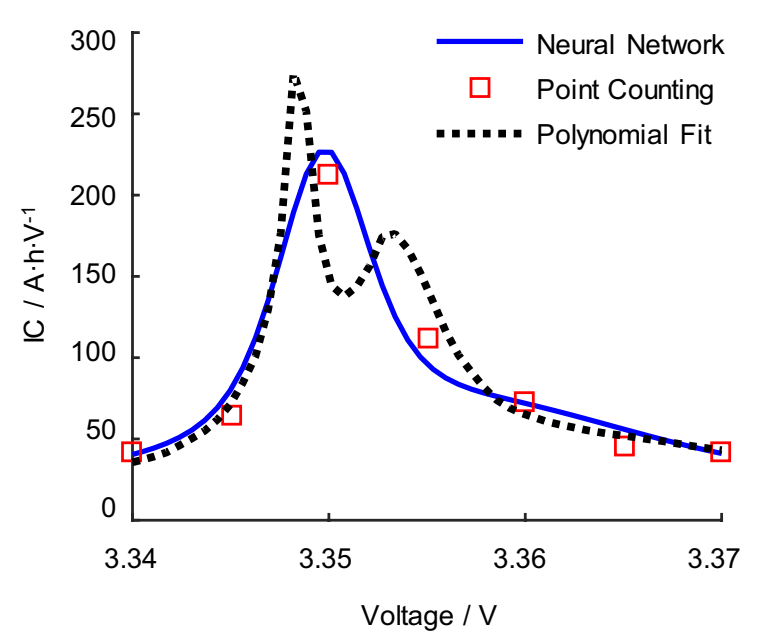

(b)

Fig. 8 Comparison of IC curves for fresh cell calculated via different methods. a Complete IC curve and $\mathbf{b}$ magnified section of the derived IC curve

The area under each peak, i.e., the capacity related to the node, can then be calculated using Eq. (10):

$Q_{i}=w_{i}^{(2)}$

In this paper, the nodes in the neural network model are sorted and labeled based on the central voltage $E_{0}$ of the voltage plateau. Therefore, from Fig. 7, it can be found that Node 5 corresponds to the voltage plateau related to phase transformation reaction (1)*II, while Nodes 3, 4 correspond to the voltage plateau related to phase transformation reaction (2) II, and Node 1 corresponds to the voltage plateau related to phase transformation reaction (5)*II. Therefore, the
IC curves do not need to be calculated since the capacities related to the voltage plateaus can be derived directly using Eq. (10) from the trained neural network model.

More specifically, the parameter $w_{5}^{(2)}$ from Node 5 of the neural network model is the capacity that corresponds to phase transformation reaction (1)*II; the parameter $w_{3}^{(2)}+w_{4}^{(2)}$ from Nodes 3, 4 is the capacity that corresponds to phase transformation reaction (2) $\mathrm{II}$; and the parameter $w_{1}^{(2)}$ from Node 1 is the capacity that corresponds to phase transformation reaction (5)*II.

The capacity evolution corresponding to different nodes is illustrated in Fig. 10. The figure shows that as the cycle time increases, the total capacity related to each phase transformation reaction decreases, which represents the LAM occurring inside the cell. Additionally, the capacity related to phase transformation reaction (1)*II decreases significantly, which represents the LLI occurring inside the cell, and it may be the main cause of the battery degradation. The analysis result is the same as that derived from the IC analysis in Sect. 4.2.

Furthermore, the capacity fade for each node can be analyzed directly and quantitatively. The capacity corresponding to reaction (1)*II decreases rapidly during the first few dozen cycles and then gradually decreases slowly. The capacity fade is affected by the cycle time, which follows a simple $t^{1 / 2}$ model, where the capacity usually fades with the degradation mechanism of LLI caused by continuous thickening of the solid electrolyte interface (SEI) film [10, 18]. Additionally, the capacities corresponding to reactions (2)*II and (5)*II show complex behavior with obvious noise and follow a linear decrement with increasing cycle time, which is usually caused by the LAM.

The parameters of each node from the trained neural network model can be used to describe the capacities corresponding to the voltage plateaus, which are also supposed to be related to the phase transformation reactions inside the cell. Therefore, this neural network model method could be used to analyze the battery aging mechanism quantitatively and diagnose the battery's $\mathrm{SOH}$.

\subsection{Brief Discussion of the Electrochemistry Mechanism Behind the Neural Network}

The above analysis shows that the neural network model proposed in this study is not a "black box" model and that the model parameters are considered with respect to certain physical meanings. This section provides a brief discussion of the electrical mechanism involved.

In most Li-ion cells, multiple electrochemical reactions occur inside the batteries. Verbrugge [50] developed theoretical equations to model the electrode voltage for multiple electrochemical reactions; the results were applied to a lithium-silicon ( $\mathrm{Li}-\mathrm{Si})$ system and showed excellent results. 


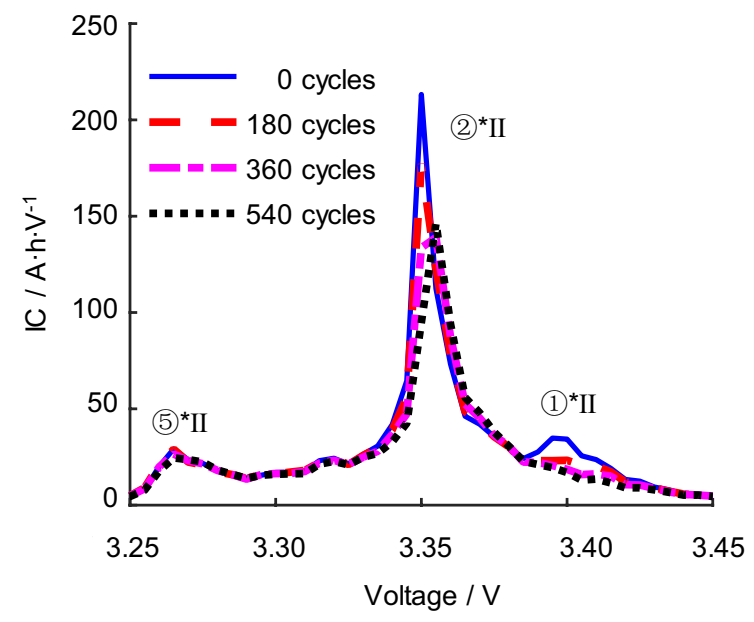

(a)

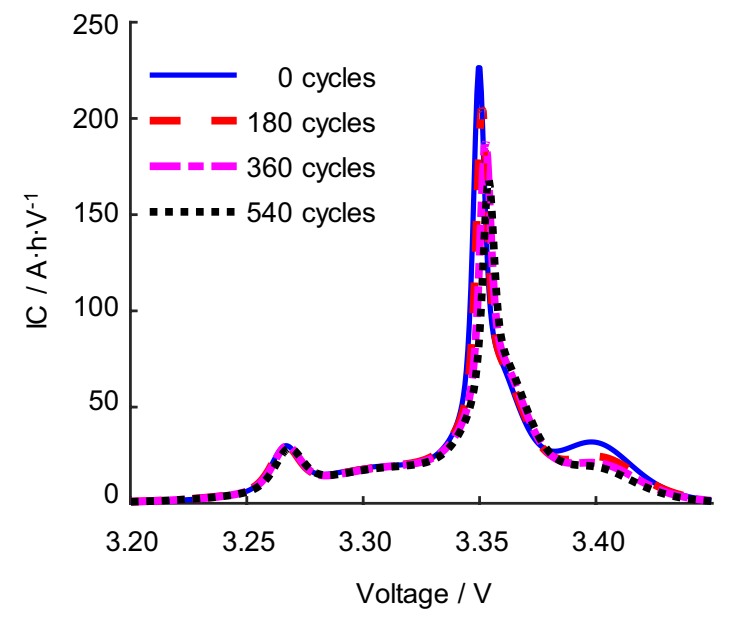

(c)

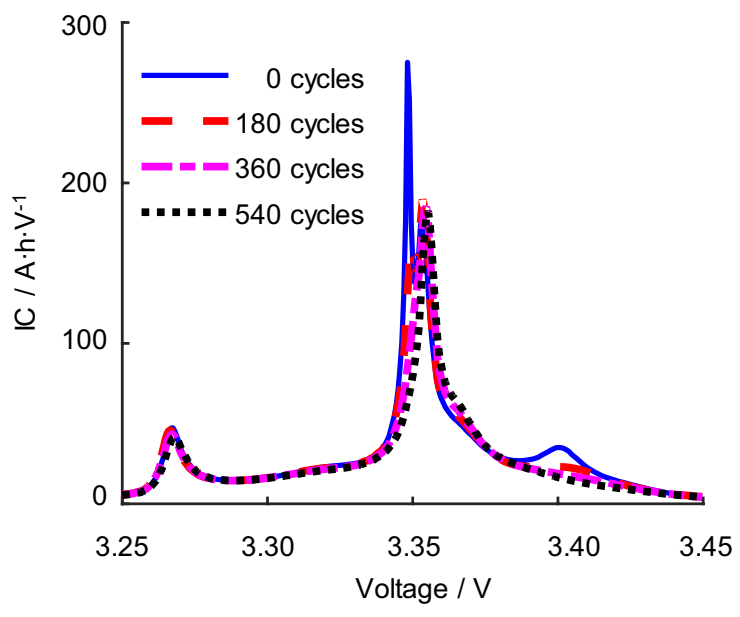

(b)

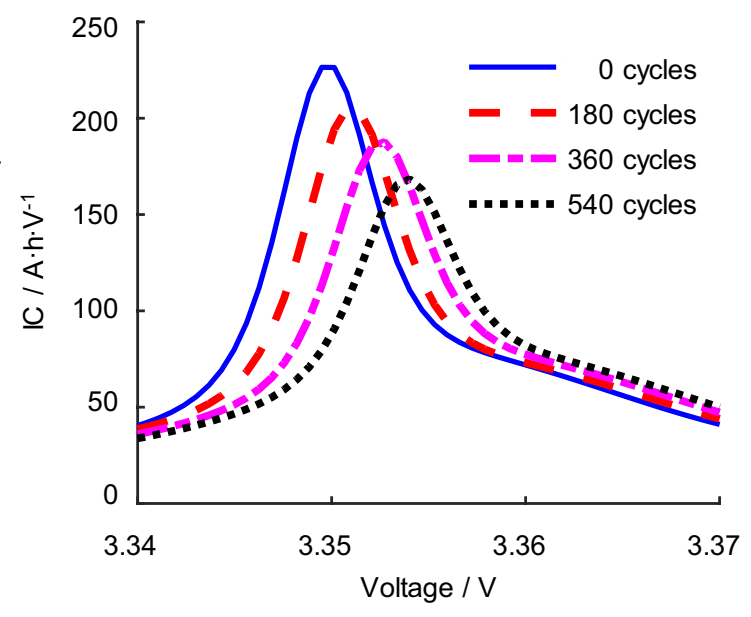

(d)

Fig. 9 IC curves calculated using different methods for cells after different numbers of cycles. a Point counting method, b polynomial curve fitting method, c neural network method, and $\mathbf{d}$ magnified section of the results calculated by the neural network method

Usually, the chemical potential describes the state of the electrode/cell as determined by the concentrations of the ionic species related to the chemical reactions. For a typical lithium insertion/deinsertion reaction, the related chemical potential $E$ follows the well-known Nernst equation, as shown in Eq. (11):

$E=E_{0}+\frac{R T}{n F} \ln \left(\frac{X-x}{x}\right)$

where $E_{0}$ is the reference potential of the electrode (specifically, the standard potential of the electrode), $F$ denotes Faraday's constant, $R$ is the universal gas constant and $T$ is the absolute temperature. Additionally, $x$ represents the fraction of the intercalated sites relative to the available sites in the host structure and $X-x$ represents the fraction of the vacant host sites. $E$ represents the electrode potential.

In most cases with low charge/discharge rates, the temperature $T, R$, and $F$ could be considered to be constants, and then, a constant $k$ could be defined as shown in Eq. (12):

$k=\frac{R T}{n F}$

Therefore, Eq. (11) could be written as Eq. (13):

$$
E=E_{0}+k \ln \left(\frac{X-x}{x}\right)
$$

Furthermore, $x$ can be calculated as shown in Eq. (14): 


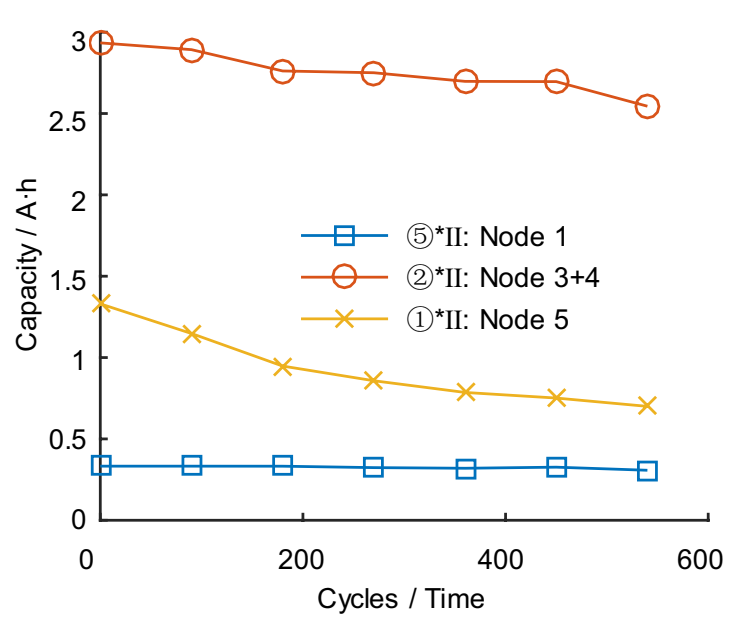

(a)

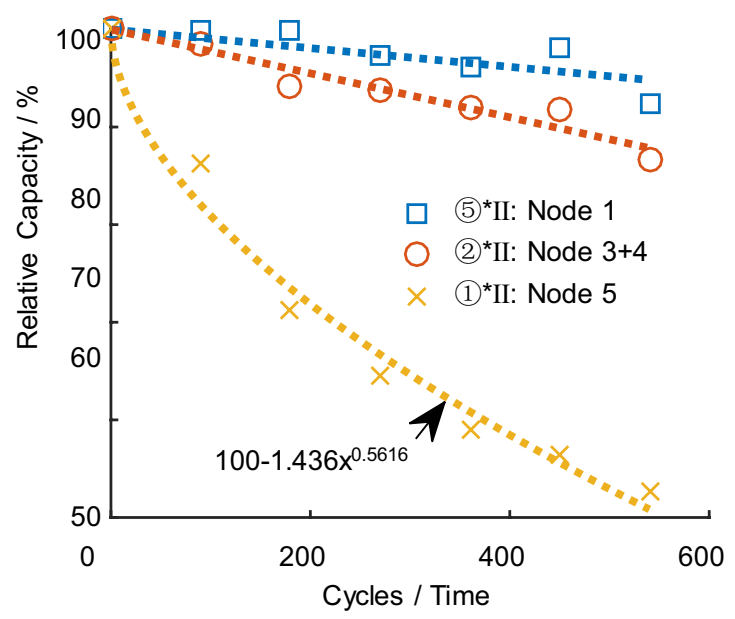

(b)

Fig. 10 Capacity evolution corresponding to the nodes in the neural network model. a Absolute capacity and $\mathbf{b}$ relative capacity

$$
x=\frac{X}{1+\mathrm{e}^{\frac{E-E_{0}}{k}}}
$$

It can be seen that Eq. (14) has the same form as the sigmoid function shown in Eq. (5).

Because more than one reaction usually occurs in the electrode, then according to Eq. (14), the $x_{i}$ related to the potential $E$ for the $i$ th reaction can be described as shown in Eq. (15):

$x_{i}=\frac{X_{i}}{1+\mathrm{e}^{\frac{E-E_{0, i}}{k_{i}}}}$

From a macroscopic perspective, the capacity $q_{i}$ that is related to a specific reaction is proportional to $x$, which means that $q_{i}$ can be given by Eq. (16):
$q_{i}=\frac{Q_{i}}{1+\mathrm{e}^{\frac{E-E_{0, i}}{k_{i}}}}$

where $Q_{i}$ represents the maximum capacity corresponding to the $i$ th reaction and $q_{i}$ represents the capacity state of the $i$ th reaction of electrode potential $E$.

The total capacity $q$ is thus dependent on the potential $E$ and can be calculated as the sum of the capacities related to the multiple electrochemical reactions as shown in Eq. (17):

$q(E)=\sum q_{i}=\sum \frac{Q_{i}}{1+\mathrm{e}^{\frac{E-E_{0, i}}{k_{i}}}}$

with

$0<q(E)<\sum Q_{i}$

A function can thus be built as shown in Eq. (18) to describe the electrode voltage with the multiple electrochemical reactions. The voltage $E$ is considered to be the independent variable, and it is used to calculate the capacity $q$. It can be seen from the above that Eq. (18) is mathematically equivalent to the neural network model described using Eqs. (4)-(10). In addition, the capacity related to the electrochemical reaction can be calculated using Eq. (10). This result shows that although the neural network method proposed in this study is intended to determine the capacities related to the voltage plateaus, it could provide a theoretical basis for the electrochemistry mechanism involved.

In this study, it is found that the proposed neural network method could be applied to the cell voltage directly to obtain the capacities related to the voltage plateaus and to analyze the aging mechanism. In theoretical terms, Eq. (18), is only suitable to describe the electrode potential. Therefore, it would be better to model the potentials of the anode and the cathode separately. However, this would complicate the calculations, and the reference electrode would have to be used to obtain the potential of each electrode, which is difficult for the real BMS in an EV to perform.

\section{Conclusions}

In this paper, several constant current charging voltage curve analysis methods, including the point counting method, the polynomial curve fitting method, and a proposed new method based on a neural network model, are compared and analyzed for the purposes of Li-ion cell aging mechanism analysis and battery SOH estimation in a BMS in an EV.

The comparison results show that the IC curves derived from the point counting method appear coarse, but the calculation involved is simple. The IC curves derived from the polynomial curve fitting method appear smooth, but the 
calculation involved is complex and there may also be overfitting problems. The IC curves that were derived from the proposed neural network model-based method enabled better analysis of the charging voltage curve, and the IC results appear to approximate the numerical derivation result more closely, while the curve shape is smooth.

In addition, the results show that the node parameters of the neural network have certain physical meanings. The capacities related to the voltage plateaus that correspond to different phase transformation reactions can easily be derived from the node parameters of the trained neural network model. The experimental results show that, based on the neural network model, the battery's aging mechanism and $\mathrm{SOH}$ can be analyzed quantitatively. The electrochemical mechanism of the neural network model is also discussed.

This method is still at the preliminary stage. Different electrochemical reactions occur in the positive electrode and the negative electrode inside the battery. To improve the model's precision and interpretability, the potentials of the positive electrode and the negative electrode should be modeled separately to obtain an improved analysis of the entire cell charging voltage curve for battery aging mechanism analysis and SOH diagnostic applications.

Acknowledgements This work is supported by the Beijing Natural Science Foundation under the Grant No.3184052, the National Natural Science Foundation of China (NSFC) under the Grant No. 51807108 and No. U1564205, and International Science and Technology Cooperation Program of China under contract No. 2016YFE0102200.

\section{Compliance with Ethical Standards}

Conflict of interest On behalf of all authors, the corresponding author states that there is no conflict of interest.

Open Access This article is distributed under the terms of the Creative Commons Attribution 4.0 International License (http://creativeco mmons.org/licenses/by/4.0/), which permits unrestricted use, distribution, and reproduction in any medium, provided you give appropriate credit to the original author(s) and the source, provide a link to the Creative Commons license, and indicate if changes were made.

\section{References}

1. Yu, C., Ji, G., Zhang, C., et al.: Cost-efficient thermal management for a $48 \mathrm{~V} \mathrm{Li}$-ion battery in a mild hybrid electric vehicle. Automot. Innov. 1(4), 320-330 (2018)

2. Zhao, B., Lv, C., Hofman, T.: Driving-cycle-aware energy management of hybrid electric vehicles using a three-dimensional markov chain model. Automot. Innov. 2, 146-156 (2019)

3. Huang, Y., Khazeraee, M., Wang, H., et al.: Design of a regenerative auxiliary power system for service vehicles. Automot. Innov. 1(1), 62-69 (2018)
4. Choi, S.S., Lim, H.S.: Factors that affect cycle-life and possible degradation mechanisms of a $\mathrm{Li}$-ion cell based on $\mathrm{LiCoO}_{2}$. J. Power Sources 111(1), 130-136 (2002)

5. Lu, L., Han, X., Li, J., et al.: A review on the key issues for lithium-ion battery management in electric vehicles. J. Power Sources 226, 272-288 (2013)

6. Xiong, R., Tian, J., Mu, H., et al.: A systematic model-based degradation behavior recognition and health monitoring method for lithium-ion batteries. Appl. Energy 207, 372-383 (2017)

7. Xiong, R., Li, L., Tian, J.: Towards a smarter battery management system: a critical review on battery state of health monitoring methods. J. Power Sources 405, 18-29 (2018)

8. Berecibar, M., Gandiaga, I., Villarreal, I., et al.: Critical review of state of health estimation methods of Li-ion batteries for real applications. Renew. Sustain. Energy Rev. 56, 572-587 (2016)

9. Bloom, I., Cole, B.W., Sohn, J.J., et al.: An accelerated calendar and cycle life study of Li-ion cells. J. Power Sources 101(2), 238-247 (2001)

10. Wang, J., Liu, P., Hicks-Garner, J., et al.: Cycle-life model for graphite-LiFePO ${ }_{4}$ cells. J. Power Sources 196(8), 3942-3948 (2011)

11. Jin, X., Vora, A., Hoshing, V., et al.: Physically-based reducedorder capacity loss model for graphite anodes in Li-ion battery cells. J. Power Sources 342, 750-761 (2017)

12. Xiong, R., Sun, F., Chen, Z., et al.: A data-driven multi-scale extended Kalman filtering based parameter and state estimation approach of lithium-ion olymer battery in electric vehicles. Appl. Energy 113, 463-476 (2014)

13. Jin, G., Matthews, D.E., Zhou, Z.: A Bayesian framework for online degradation assessment and residual life prediction of secondary batteries inspacecraft. Reliab. Eng. Syst. Saf. 113, 7-20 (2013)

14. Wang, Y., Yang, D., Zhang, X., et al.: Probability based remaining capacity estimation using data-driven and neural network model. J. Power Sources 315, 199-208 (2016)

15. Zheng, Y., Qin, C., Lai, X., et al.: A novel capacity estimation method for lithium-ion batteries using fusion estimation of charging curve sections and discrete Arrhenius aging model. Appl. Energy 251, 113327 (2019)

16. Han, X., Ouyang, M., Lu, L., et al.: A comparative study of commercial lithium ion battery cycle life in electric vehicle: capacity loss estimation. J. Power Sources 268, 658-669 (2014)

17. Birkl, C.R., Roberts, M.R., McTurk, E., et al.: Degradation diagnostics for lithium ion cells. J. Power Sources 341, 373-386 (2017)

18. Vetter, J., Novák, P., Wagner, M.R., et al.: Ageing mechanisms in lithium-ion batteries. J. Power Sources 147(1-2), 269-281 (2005)

19. Dubarry, M., Truchot, C., Liaw, B.Y.: Synthesize battery degradation modes via a diagnostic and prognostic model. J. Power Sources 219, 204-216 (2012)

20. Han, X., Ouyang, M., Lu, L., et al.: A comparative study of commercial lithium ion battery cycle life in electrical vehicle: aging mechanism identification. J. Power Sources 251, 38-54 (2014)

21. Han, X., Ouyang, M., Lu, L., et al.: Cycle life of commercial lithium-ion batteries with lithium titanium oxide anodes in electric vehicles. Energies 7(8), 4895-4909 (2014)

22. Zheng, Y., Wang, J., Qin, C., et al.: A novel capacity estimation method based on charging curve sections for lithium-ion batteries in electric vehicles. Energy 185, 361-371 (2019)

23. Hu, X., Li, S.E., Jia, Z., et al.: Enhanced sample entropy-based health management of Li-ion battery for electrified vehicles. Energy 64, 953-960 (2014)

24. Mai, L., Yan, M., Zhao, Y.: Track batteries degrading in real time. Nature 546(7659), 469-470 (2017) 
25. Dubarry, M., Truchot, C., Cugnet, M., et al.: Evaluation of commercial lithium-ion cells based on composite positive electrode for plug-in hybrid electric vehicle applications. Part I: initial characterizations. J. Power Sources 196(23), 10328-10335 (2011)

26. He, Y., Shen, J.F., Shen, J.N., et al.: Embedding monotonicity in the construction of polynomial open-circuit voltage model for lithium-ion batteries: a semi-infinite programming formulation approach. Ind. Eng. Chem. Res. 54(12), 3167-3174 (2015)

27. Weng, C., Sun, J., Peng, H.: A unified open-circuit-voltage model of lithium-ion batteries for state-of-charge estimation and stateof-health monitoring. J. Power Sources 258, 228-237 (2014)

28. Sun, F., Xiong, R.: A novel dual-scale cell state-of-charge estimation approach for series-connected battery pack used in electric vehicles. J. Power Sources 274, 582-594 (2015)

29. Li, Y., Abdel-Monem, M., Gopalakrishnan, R., et al.: A quick on-line state of health estimation method for Li-ion battery with incremental capacity curves processed by Gaussian filter. J. Power Sources 373, 40-53 (2018)

30. Pastor-Fernández, C., Uddin, K., Chouchelamane, G.H., et al.: A comparison between electrochemical impedance spectroscopy and incremental capacity-differential voltage as Li-ion diagnostic techniques to identify and quantify the effects of degradation modes within battery management systems. J. Power Sources 360, 301-318 (2017)

31. Dubarry, M., Liaw, B.Y.: Identify capacity fading mechanism in a commercial $\mathrm{LiFePO}_{4}$ cell. J. Power Sources 194(1), 541-549 (2009)

32. Weng, C., Cui, Y., Sun, J., et al.: On-board state of health monitoring of lithium-ion batteries using incremental capacity analysis with support vector regression. J. Power Sources 235, 36-44 (2013)

33. Safari, M., Delacourt, C.: Aging of a commercial graphite/ $\mathrm{LiFePO}_{4}$ cell. J. Electrochem. Soc. 158(10), A1123-A1135 (2011)

34. Bloom, I., Jansen, A.N., Abraham, D.P., et al.: Differential voltage analyses of high-power, lithium-ion cells: 1 . Technique and application. J. Power Sources 139(1-2), 295-303 (2005)

35. Bloom, I., Walker, L.K., Basco, J.K., et al.: Differential voltage analyses of high-power lithium-ion cells. 4. Cells containing NMC. J. Power Sources 195(3), 877-882 (2010)

36. Honkura, K., Takahashi, K., Horiba, T.: Capacity-fading prediction of lithium-ion batteries based on discharge curves analysis. J. Power Sources 196(23), 10141-10147 (2011)

37. Dahn, H.M., Smith, A.J., Burns, J.C., et al.: User-friendly differential voltage analysis freeware for the analysis of degradation mechanisms in Li-ion batteries. J. Electrochem. Soc. 159(9), A1405-A1409 (2012)

38. Feng, X., Li, J., Ouyang, M., et al.: Using probability density function to evaluate the state of health of lithium-ion batteries. J. Power Sources 232, 209-218 (2013)

39. Zheng, Y., Ouyang, M., Han, X., et al.: Investigating the error sources of the online state of charge estimation methods for lithium-ion batteries in electric vehicles. J. Power Sources 377, 161-188 (2018)

40. Prada, E., Di Domenico, D., Creff, Y., et al.: Simplified electrochemical and thermal model of $\mathrm{LiFePO}_{4}$-Graphite Li-ion batteries for fast charge applications. J. Electrochem. Soc. 159(9), A1508-A1519 (2012)

41. Kassem, M., Bernard, J., Revel, R., et al.: Calendar aging of a graphite $/ \mathrm{LiFePO}_{4}$ cell. J. Power Sources 208, 296-305 (2012)

42. Weng, C., Feng, X., Sun, J., et al.: State-of-health monitoring of lithium-ion battery modules and packs via incremental capacity peak tracking. Appl. Energy 180, 360-368 (2016)

43. Chan, C.C., Lo, E.W.C., Weixiang, S.: The available capacity computation model based on artificial neural network for leadacid batteries in electric vehicles. J. Power Sources 87(1-2), 201-204 (2000)

44. Weigert, T., Tian, Q., Lian, K.: State-of-charge prediction of batteries and battery-supercapacitor hybrids using artificial neural networks. J. Power Sources 196(8), 4061-4066 (2011)

45. You, G., Park, S., Oh, D.: Real-time state-of-health estimation for electric vehicle batteries: a data-driven approach. Appl. Energy 176, 92-103 (2016)

46. Bai, G., Wang, P., Hu, C., et al.: A generic model-free approach for lithium-ion battery health management. Appl. Energy 135, 247-260 (2014)

47. Kang, L., Zhao, X., Ma, J.: A new neural network model for the state-of-charge estimation in the battery degradation process. Appl. Energy 121, 20-27 (2014)

48. Wu, J., Zhang, C., Chen, Z.: An online method for lithium-ion battery remaining useful life estimation using importance sampling and neural networks. Appl. Energy 173, 134-140 (2016)

49. Kassem, M., Delacourt, C.: Postmortem analysis of calendar-aged graphite/LiFePO 4 cells. J. Power Sources 235, 159-171 (2013)

50. Verbrugge, M., Baker, D., Xiao, X.: Formulation for the treatment of multiple electrochemical reactions and associated speciation for the Lithium-Silicon electrode. J. Electrochem. Soc. 163(2), A262-A271 (2016) 